\title{
Immunologic Aging in Adults With Congenital Heart Disease: Does Infant Sternotomy Matter?
}

\author{
Robert W. Elder, MD ${ }^{a}$, Roshan P. George, MD $^{b}$, Nancy M. McCabe, BA, BSN ${ }^{c}$, Fred H. \\ Rodriguez III, MD ${ }^{d}$, Wendy M. Book, MD ${ }^{e}$, William T. Mahle, MD ${ }^{d}$, and Allan D. Kirk, MD, PhD ${ }^{f}$ \\ aSection of Cardiology, Department of Pediatrics and Internal Medicine, Yale University School of \\ Medicine, PO Box 208064; 333 Cedar Street, 3 LCI, New Haven, CT 06510 \\ bDepartment of Pediatric Nephrology, Emory University School of Medicine, 1405 Clifton Road, \\ NE, Atlanta, GA 30322 \\ cNell Hodgson Woodruff School of Nursing, Emory University, 1520 Clifton Rd NE, Atlanta, GA \\ 30322 \\ dDivision of Pediatric Cardiology, Sibley Heart Center, Children's Healthcare of Atlanta, Emory \\ University School of Medicine, 1405 Clifton Road, NE, Atlanta, GA 30322 \\ eDepartment of Internal Medicine, Division of Cardiology, Emory University School of Medicine, \\ 1365 Clifton Road Northeast, Suite A2447, Atlanta, GA 30322 \\ fDepartment of Surgery, Duke University School of Medicine, DUMC 3704, Durham, NC 27710
}

\begin{abstract}
Thymectomy is performed routinely in infants undergoing cardiothoracic surgery. Children poststernotomy have decreased numbers of T-lymphocytes, although the mechanisms involved and long-term consequences of this have not been defined. We hypothesized that lymphopenia in patients with adult congenital heart disease (ACHD) would be reflective of premature $\mathrm{T}$ cell maturation and exhaustion. Adults with ACHD who had sternotomy to repair congenital heart disease as infants ( $<1$ year) and age-matched ACHD patients without prior sternotomy were studied using polychromatic flow cytometry interrogating markers of lymphocyte maturation, exhaustion and senescence. Group differences were analyzed using Mann-Whitney-U and Fisher's exact tests. 18 ACHD patients ages $21-40$ years participated: 10 cases and 8 controls. Median age at sternotomy for cases was 52 days. Cases and controls were matched for age ( 28.9 vs. 29.1 years; $\mathrm{p}=0.83$ ), gender $(\mathrm{p}=0.15)$, race $(\mathrm{p}=0.62)$, and had similar case complexity. Cases had a lower mean percentage of cytotoxic CD8 lymphocytes compared to controls (26.8 vs. 33.9\%; $\mathrm{p}=0.016$ ), with fewer naive, undifferentiated CD8 T-cells ( 31.0 vs. $53.6 \%$; $\mathrm{p}=0.027$ ). CD8 cells expressing PD1, a marker of immune exhaustion, trended higher in cases vs. controls (25.6 vs. $19.0 \% ; \mathrm{p}=0.083$ ). Mean percentage of CD4 cells was higher in cases vs. controls (65.6 vs. 59.6\%; $\mathrm{p}=0.027$ ), without differences in CD4 T-cell maturation subtype. In summary, ACHD patients who undergo sternotomy as infants exhibit differences in T lymphocyte composition compared to
\end{abstract}

Author for correspondence: Robert W Elder, MD, Yale University School of Medicine, P.O. Box 208064, 333 Cedar Street, 3 LCI, New Haven, CT 06510, Telephone: 203-785-2022, Fax: 203-737-2786, robert.elder@yale.edu. 
ACHD controls, suggesting accelerated immunologic exhaustion. Investigation is warranted to assess the progressive nature and clinical impact of this immune phenotypic change.

\section{Keywords}

Congenital heart disease; surgery; adult congenital heart disease; thymectomy; immune function

\section{Introduction}

The thymus is the major site of production for $\mathrm{T}$ cells in infants and plays an important role in immune education[1]. This large gland obscures the cardiothoracic operative field in infants and is routinely removed and destroyed at the time of sternotomy to enable repair of congenital heart disease. The consequences of thymic destruction in infants requiring sternotomy are not well defined[2]. Prior limited work examining immune function in children who required sternotomy as infants suggests alterations in $\mathrm{T}$ cell populations and response to antigen, though not to a level that has been associated with immune dysfunction over a short period of follow-up[3,4].

In the absence of thymopoiesis, $\mathrm{T}$ cell homeostasis is maintained through peripheral activation and division[5]. The consequences of this manifest as advanced $\mathrm{T}$ cell maturation; specifically, increased terminal differentiation, progressive activation induced cell death and eventual loss of repertoire diversity[6]. As the thymus undergoes normal atrophy with age, this immune phenotype has been shown to be related to age-induced immune dysfunction in the general population, and has been linked to a number of clinical concerns including increased susceptibility to viral infections and reactivations, higher rates of auto-immunity, and reduced immune surveillance of cancer[7,8]. The process of peripheral $\mathrm{T}$ cell homeostasis is gradual and unfolds over years[9].

With the majority of children with congenital heart disease now surviving into adulthood[10], it is reasonable to anticipate that the consequences of thymectomy are only now becoming perceptible, and long-term immune function could be of concern. We therefore have studied adult congenital heart disease (ACHD) patients who have had their thymus removed as infants to examine immunological differences that could be reflective of the consequences of progressive, extrathymic $\mathrm{T}$ cell homeostasis compared to a control population who never required sternotomy.

\section{Material and methods}

A prospective, case control study was performed. ACHD patients greater than 18 years were recruited who had required a sternotomy at less than one year of age to repair congenital heart disease (CHD). Data regarding CHD and surgical history were collected by chart review. Although operative notes were not consistently available for all subjects, institutional practice was complete thymus removal at the time of sternotomy to enable visualization and cannulation of the heart for repair. Control ACHD patients who had never required sternotomy, but were followed in ACHD clinic, were recruited and matched for age. Gender 
and race demographics were also collected. All patients signed informed consent and approval was obtained by the IRB at Emory University prior to commencement of the study.

Exclusion criteria included patients with known or suspected DiGeorge syndrome (22q11 deletion). This included ACHD patients with conotruncal lesions typically associated with DiGeorge (e.g. tetralogy of Fallot, truncus arteriosus, interrupted aortic arch) unless there was genetic testing documenting a normal result. Other exclusionary criteria related to conditions with possible effects on immune function and included current pregnancy, serious infection requiring hospitalization or medication within the prior 3 months, or NYHA class III-IV.

Peripheral blood was collected in Cyto-chex BCT tubes (Streck, Omaha, NE) and analyzed using polychromatic flow cytometry as previously described[11]. Briefly, flow cytometry was performed to assess $\mathrm{T}$ cell sub-type including $\mathrm{CD}^{+}$(all $\mathrm{T}$ cells), $\mathrm{CD}^{+} \mathrm{CD}^{+}$(helper $\mathrm{T}$ cells), $\mathrm{CD}^{+} \mathrm{CD}^{+}$(cytotoxic $\mathrm{T}$ cells), and determinants of $\mathrm{T}$ cell maturation status including naive (CD45RA+/CCR7+), central memory (CD45RA-/CCR7+), effective memory (CD45RA-/CCR7-), and T-effector memory cells expressing CD45+ RA (TEMRA) lymphocytes (CD45RA+/CCR7)[12]. In addition, we assessed markers typically associated with lymphocyte exhaustion and senescence in the CD8 subset: PD1+ for exhaustion, CD57+ for senescence, CD27- and CD28- for senescence[13,14].

Differences in demographic and immunological variables between ACHD patients with and without infant sternotomy were analyzed using Mann-Whitney-U and Fisher's exact tests. A one-way multivariate analysis of variance (MANOVA) was used to determine differences between the groups with and without sternotomy on all four cell types (naive, central memory, effective memory, and TEMRA) both CD8 and CD4 types, simultaneously. All data was analyzed using SPSS v.20. An alpha level of 0.05 was considered statistically significant.

\section{Results}

A total of 18 patients were recruited, including 10 who underwent CHD surgery requiring sternotomy at a median age of 52.2 days (range 1-330 days). The baseline data for the two groups is presented in Table I. The groups were well matched for age, gender, and race.

Diagnoses for the two groups of patients are listed in Table II. Although there was no way to match diagnoses, in both groups there was a mix of simple, moderate, and complex lesions. One patient in the study group underwent CHD surgery prior to sternotomy (shunt) and 4 of the patients had further cardiac surgical interventions after initial sternotomy. Six of the 8 patients in the control group required surgical or cardiac catheterization interventions: pacemaker (3), coarctation stenting (1), PDA ligation via thoracotomy (1), and balloon mitral valvuloplasty (1).

The mean percentage of total CD8+ T cells was lower in the sternotomy group as compared to control (26.8 vs. $33.9 \%, U=13.0, \mathrm{p}=0.016, \mathrm{r}=-.57$ ). Accordingly, the percentage of CD4+ $\mathrm{T}$ cells was reciprocally higher in sternotomy vs. controls ( 65.5 vs. $59.6 \%, U=15.0, \mathrm{p}=0.027$, 
$\mathrm{r}=-.52$ ). This was reflected in a significant difference between the mean CD4 to CD8 ratio of the cell types: 2.8 in cases vs. 1.8 in controls $(U=11.0, \mathrm{p}=0.009, \mathrm{r}=-.61$.)

Mean percentage of CD4 and CD8 T cell subtypes, including naive, central memory, effector memory, and TEMRA cells are depicted (Figure 1A and B). Naive CD8 T cells were lower in sternotomy subjects as compared to controls ( 31 vs. $53.6 \%, U=15.0, \mathrm{p}=0.027, \mathrm{r}=-.52$ ), with a concomitant increase in cells expressing markers of advanced differentiation. Percentage of naive CD4 T cells was less than controls, though this difference did not reach statistical significance ( 25.4 vs. $36.3 \%, U=26.0, \mathrm{p}=0.24$ ). In general, the sternotomy group tended to skew towards mature cell types, though only the percentage of naive CD8 cell type was significant.

We used one-way multivariate analysis of variance to examine differences between cell types for both CD4 and CD8. For CD4 cell types, no differences were found between persons with and without a sternotomy on all four simultaneously $(\mathrm{F}(290)=0.89, \mathrm{p}=0.50$, Wilks' $\lambda=0.27$ ). However, for CD8 cell types a trending difference was found between persons with and without a sternotomy on all four simultaneously $(F(1823)=2.75, p=0.07$, Wilks' $\lambda=0.54)$.

Finally, we measured known markers of cellular immune senescence. PD1, an inhibitory receptor expressed on $\mathrm{T}$ cells following activation or antigen experience and typically considered as a sign of T cell exhaustion, was elevated as compared to controls and trended toward significance ( 25.6 vs. $19 \%, U=20.0, \mathrm{p}=0.08, \mathrm{r}=-0.42$ ). Overall, markers of senescence on CD8 $\mathrm{T}$ cells tended to be higher in the sternotomy group, but these differences were not significant (Figure 2).

\section{Discussion}

Congenital heart surgery has undergone revolutionary advancement over the past several decades, with long-term survival becoming increasingly commonplace[10]. While early congenital defect repair was focused on short-term technical success, the modern congenital heart surgeon has the luxury and responsibility to consider the consequences of the operative approach, with the expectation that their patients will live into adulthood. While it is clear that thymectomy improves the operative approach and has few immediate immune effects, cognizance of the more protracted consequences of this maneuver are now relevant.

In this pilot study, we examined the $\mathrm{T}$ cell repertoire of adults who had undergone sternotomy as infants, paying particular attention to phenotypic changes known to be associated with thymic atrophy and peripheral $\mathrm{T}$ cell homeostatic activation. We note significant differences in the $\mathrm{T}$ cell repertoire as compared to age and gender-matched controls with heart disease. To our knowledge, this is the first study to examine such differences in an adult population of patients with CHD. They are perhaps a bellwether of emerging chronic immune consequences of routine thymectomy that warrant examination as CHD survivors grow more commonplace.

It is known that $\mathrm{T}$ cells perform essential activities both in mediating host defense and in establishing a regulatory process to avoid autoimmunity[7,8,15]. With age, a breakdown in 
these functions has been clearly related to decreased thymic function and manifest as increased reactivation of latent herpes viruses (e.g. zoster) and progressive risk of $\mathrm{T}$ cell mediated autoimmunity. CD8 positive $\mathrm{T}$ cells, in particular, have less intrinsic regenerative capacity, and through their continuous deployment to kill host cells infected with pathogens are more susceptible to the consequences of a limited source of new thymic emigrants.

The concept of immune aging in elderly patients is well established[7,15]. The most noticeable age associated changes in $\mathrm{T}$ cell immunity are decreased numbers of naive $\mathrm{T}$ cells and increased numbers of memory $\mathrm{T}$ cells[15]. Indeed, naive $\mathrm{T}$ cells are thought to have the most robust lymphoproliferative capacity and their abundance is seen as a marker of a "young" immune system. Numerous other immune alterations have been reported, including changes in $\mathrm{T}$ cell proliferation, cytokine production, memory response, and cytotoxicity[15].

As early as 1987, Brearley et al. examined 18 children aged 9 mo-3 years who underwent young sternotomy at less than 3 months[2]. Thymectomized children had lower numbers of $\mathrm{T}$ cells and $\mathrm{T}$ cell sub-types as compared to controls, in addition to decreased response to antigen assays. Although the levels did not seem to reach clinical significance, they concluded "routine thymectomy in paediatric cardiac surgery should be avoided."

Wells and colleagues looked at 25 patients who had neonatal surgery requiring sternotomy at less than 30 days[3]. After 1 year, patients showed a significantly decreased percentage of all T lymphocytes and specifically CD4 cell types, although of interest there were no differences in CD8 $\mathrm{T}$ cells. The results did not approach the critical levels thought to be important in immunodeficiency. In a follow up study examining children with an average age of 10 years who had thymectomy at a mean age of 2.6 months, another study similarly found lower counts of total T lymphocytes, and specifically CD4 T lymphocytes, in the sternotomy group[4]. Neither of these studies focused on the different subtypes of $\mathrm{T}$ lymphocytes, including naive or memory populations, or markers of immune exhaustion or senescence.

Our population of ACHD patients, whose mean age was 28.9 years, demonstrated differences in the $\mathrm{T}$ cell repertoire as compared to age and gender-matched controls with CHD but without thymectomy. Sternotomy patients tended to have lower levels of naive T cells and more memory cells, consistent with a known immunologic aging phenotype. These patients also tended to have increased evidence of $\mathrm{T}$ lymphocyte exhaustion but not senescence, suggesting that age per se was not an issue so much as hyper-utility in the absence of thymic-derived repopulation.

Although these differences are preliminary, the concept of immune aging post-sternotomy is biologically plausible and fits with prior work[16]. Indeed, if any group were at risk for immune dysfunction post infant sternotomy, logically it would seem to be an adult population. It is well known that the thymus gland is the major site of production for $\mathrm{T}$ cells. Immature cells must undergo a complicated selection process of defining optimal affinity for major histocompatibility (MHC) while preventing recognition of self. Some of this selection happens in the prenatal period, but the process is thought to continue well after birth. Indeed, in many infants the thymus at the time of CHD surgery is quite large (Figure 3). 
At most cardiac centers, the standard surgical approach is complete removal of the thymus at the time of infant sternotomy, which is either sent to the pathology laboratory or destroyed. Removal enables complete visualization of the surgical field and cannulation of the heart for bypass. It is possible that age at surgery could have important differences. For this study, we considered all patients who had been thymectomized less than 1 year, but a 3-day-old patient undergoing arterial switch operation may be quite different from an immune perspective than an 11-month-old who requires surgical aortic valvuloplasty.

There are a number of potential implications for the population of ACHD patients. Recent preliminary data has suggested an increased prevalence of cancer in ACHD patients as compared to the general population (personal communication, Dr. Michelle Gurvitz). While this is preliminary and almost certainly multi-factorial (e.g. radiation exposure, genetic predisposition, etc.), the role of $\mathrm{T}$ cell function should not be discounted. ACHD patients are at risk for a variety of infections and auto-immune disease, although this has not be shown to be elevated as compared to controls. T cells might play a role in allergic response or rejection after transplant. Clearly, although the differences described in this manuscript do not equate to clinical effects, the concept merits further thought and research.

\section{Limitations}

This study has a number of limitations. This is a pilot study and as such the numbers in each group are small, making the statistical power less. Thymectomy at the time of sternotomy is assumed as we did not have access to all original surgical reports and some reports did not include details regarding the fate of the thymus, although thymectomy was the standard surgical practice at our institution. Although we compared percentages of different cell types and makers, we did not have data on actual cell numbers. This study did not include a functional component such as response to a given antigen.

In summary, ACHD patients with infant sternotomy show differences in T cell repertoire. Compared to age matched controls, they have less naive $\mathrm{T}$ cells and some signs of increased immune aging that are consistent with an elderly population of patients. Whether this is clinically significant remains unknown. Further research is needed to determine the longterm clinical significance.

\section{References}

1. Ventevogel MS, Sempowski GD. Thymic rejuvenation and aging. Current opinion in immunology. 2013; 25:516-522. [PubMed: 23831111]

2. Brearley S, Gentle TA, Baynham MI, Roberts KD, Abrams LD, Thompson RA. Immunodeficiency following neonatal thymectomy in man. Clinical and experimental immunology. 1987; 70:322-327. [PubMed: 3427824]

3. Wells WJ, Parkman R, Smogorzewska E, Barr M. Neonatal thymectomy: does it affect immune function? The Journal of thoracic and cardiovascular surgery. 1998; 115:1041-1046. [PubMed: 9605073]

4. Eysteinsdottir JH, Freysdottir J, Haraldsson A, Stefansdottir J, Skaftadottir I, Helgason H, Ogmundsdottir HM. The influence of partial or total thymectomy during open heart surgery in infants on the immune function later in life. Clinical and experimental immunology. 2004; 136:349355. [PubMed: 15086401] 
5. Williams KM, Hakim FT, Gress RE. T cell immune reconstitution following lymphodepletion. Seminars in immunology. 2007; 19:318-330. [PubMed: 18023361]

6. Goronzy JJ, Weyand CM. T cell development and receptor diversity during aging. Current opinion in immunology. 2005; 17:468-475. [PubMed: 16098723]

7. Goronzy JJ, Weyand CM. Understanding immunosenescence to improve responses to vaccines. Nature immunology. 2013; 14:428-436. [PubMed: 23598398]

8. Prelog M. Aging of the immune system: a risk factor for autoimmunity? Autoimmunity reviews. 2006; 5:136-139. [PubMed: 16431345]

9. Chen J, Li J, Lim FC, Wu Q, Douek DC, Scott DK, Ravussin E, Hsu HC, Jazwinski SM, Mountz JD. Louisiana Healthy Aging S. Maintenance of naive CD8 T cells in nonagenarians by leptin, IGFBP3 and T3. Mechanisms of ageing and development. 2010; 131:29-37. [PubMed: 19941883]

10. Marelli AJ, Mackie AS, Ionescu-Ittu R, Rahme E, Pilote L. Congenital heart disease in the general population: changing prevalence and age distribution. Circulation. 2007; 115:163-172. [PubMed: 17210844]

11. Pearl JP, Parris J, Hale DA, Hoffmann SC, Bernstein WB, McCoy KL, Swanson SJ, Mannon RB, Roederer M, Kirk AD. Immunocompetent T-cells with a memory-like phenotype are the dominant cell type following antibody-mediated T-cell depletion. American journal of transplantation: official journal of the American Society of Transplantation and the American Society of Transplant Surgeons. 2005; 5:465-474.

12. Sallusto F, Lenig D, Forster R, Lipp M, Lanzavecchia A. Two subsets of memory T lymphocytes with distinct homing potentials and effector functions. Nature. 1999; 401:708-712. [PubMed: 10537110]

13. Strioga M, Pasukoniene V, Characiejus D. CD8+CD28- and CD8+ CD57+ T cells and their role in health and disease. Immunology. 2011; 134:17-32. [PubMed: 21711350]

14. Youngblood B, Wherry EJ, Ahmed R. Acquired transcriptional programming in functional and exhausted virus-specific CD8 T cells. Current opinion in HIV and AIDS. 2012; 7:50-57. [PubMed: 22134341]

15. Lee N, Shin MS, Kang I. T-cell biology in aging, with a focus on lung disease. The journals of gerontology Series A, Biological sciences and medical sciences. 2012; 67:254-263.

16. Halnon NJ, Cooper P, Chen DY, Boechat MI, Uittenbogaart CH. Immune dysregulation after cardiothoracic surgery and incidental thymectomy: maintenance of regulatory $\mathrm{T}$ cells despite impaired thymopoiesis. Clinical \& developmental immunology. 2011; 2011:915864. [PubMed: 21776289] 
CD4 Cell Subtype

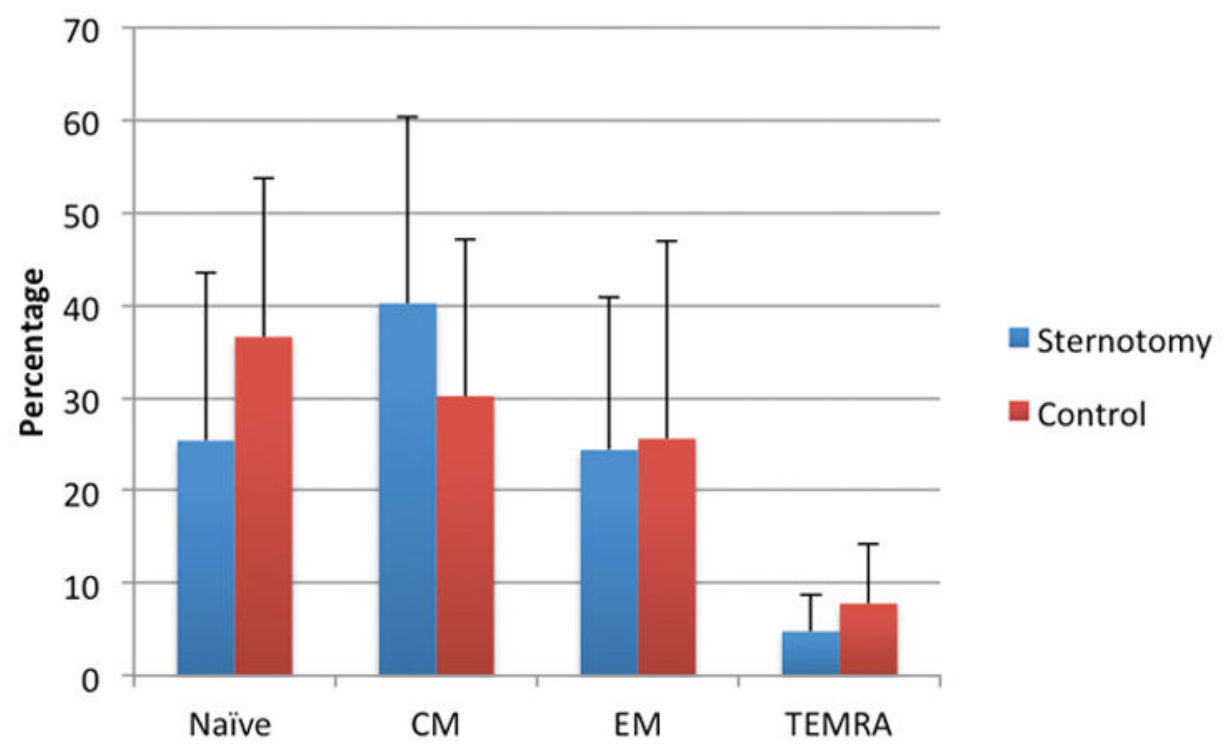

CD8 Cell Subtype

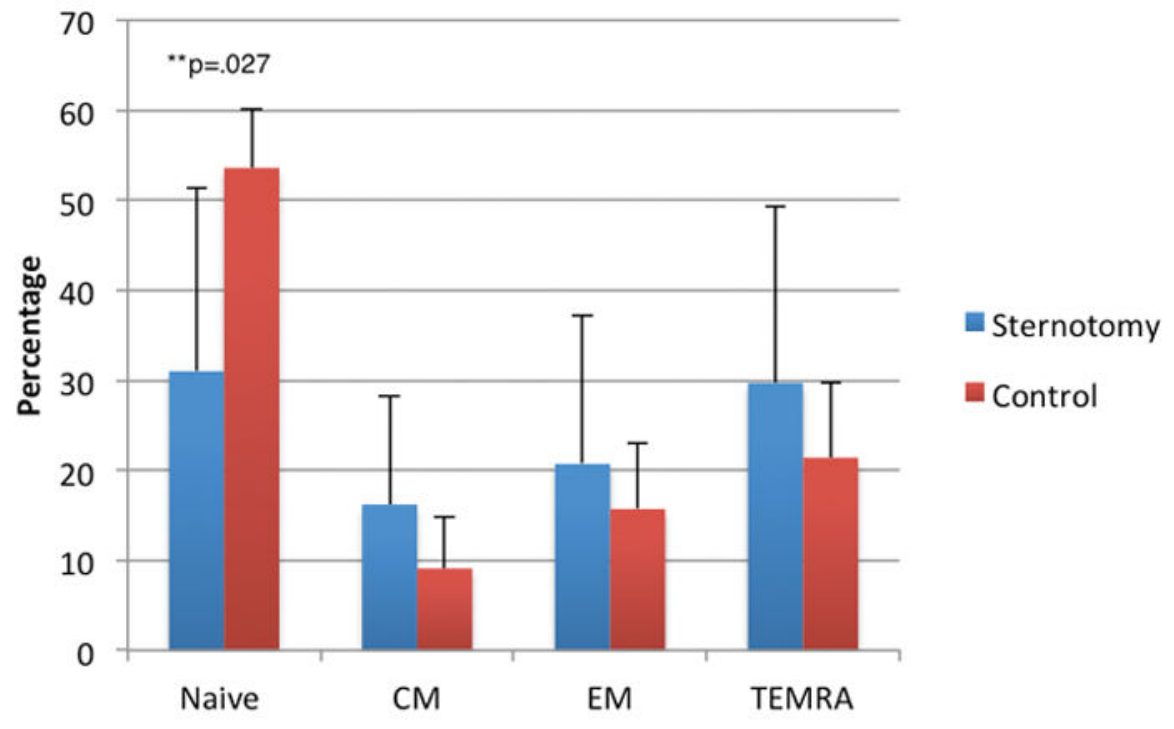

Figure 1A and $1 \mathrm{~B}$.

Percentage of T lymphocyte populations of different maturation. A. Sternotomy patients were similar to controls in regard to CD4+ T cells, though tended to have fewer naïve cells. B. Sternotomy patients had statistically fewer naive CD8+ cells than controls. Other cell populations were not statistically different between cases and controls. Cells along the $\mathrm{x}$-axis 
are displayed in order of maturation. $\mathrm{CM}=$ central memory. EM=effective memory. TEMRA=T-effector memory cells expressing CD45+ RA (TEMRA) 


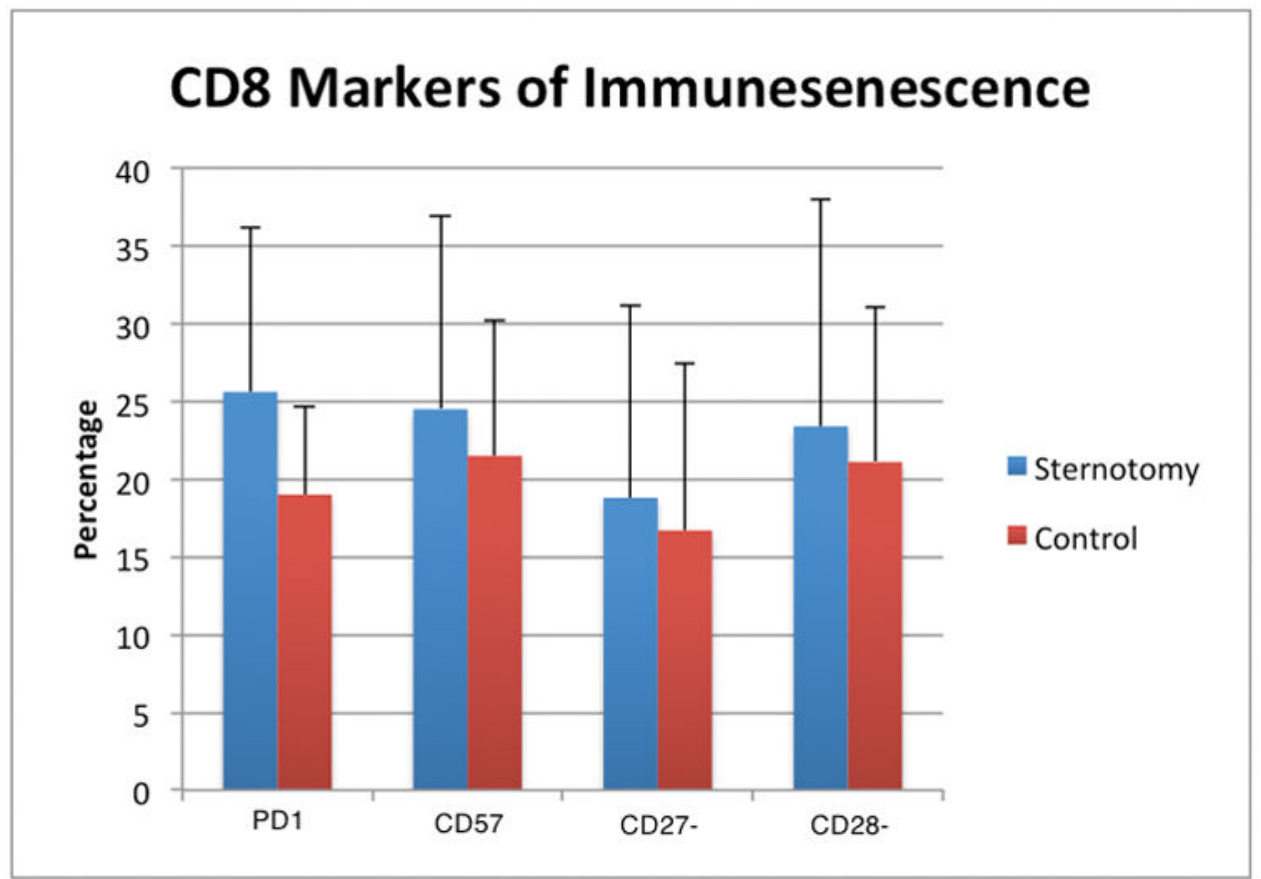

Figure 2.

Differences between sternotomy and control group for known markers of lymphocyte exhaustion and senescence in the CD8 subset: PD1+ for exhaustion, CD57+ for senescence, CD27- and CD28- for senescence. 


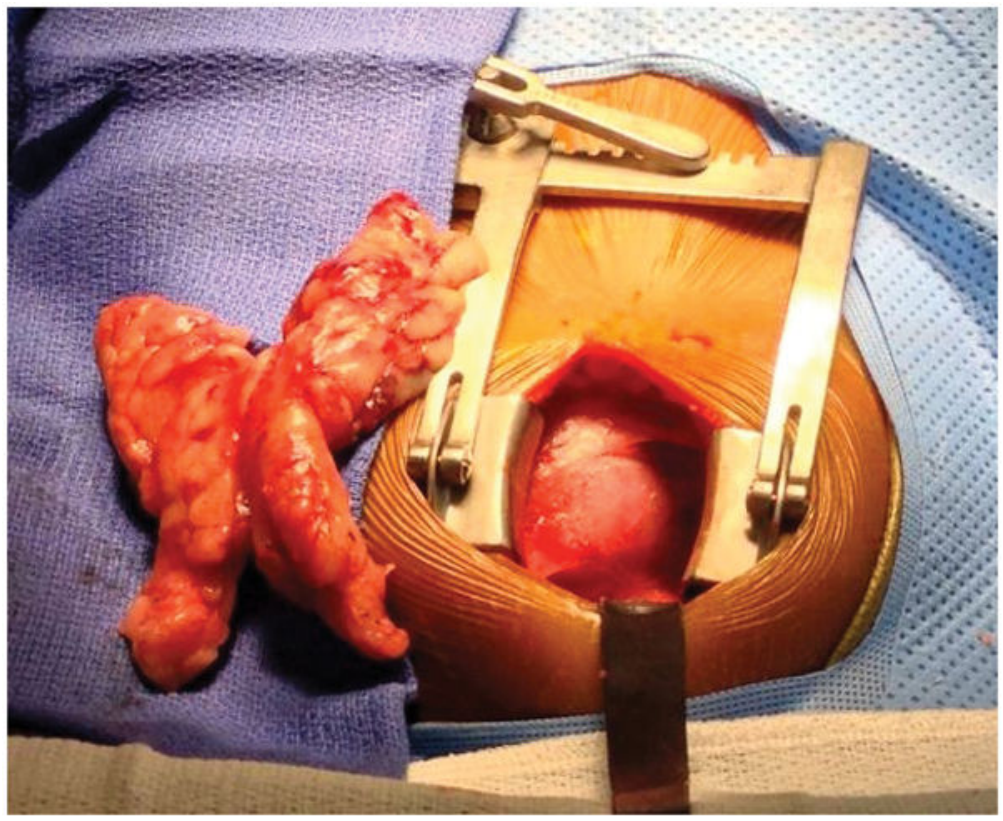

Figure 3.

Intraoperative photograph of a 3-month-old infant with congenital heart disease at the time of surgical repair, with large thymus dissected out and removed (left) to enable complete visualization and repair of the heart. 


\section{Table I}

Patient Population

Demographics of the patient population for sternotomy versus control groups.

\begin{tabular}{|llll|}
\hline & Sternotomy & Control & Sig. \\
\hline Mean age at sternotomy & 85.5 days & NA & NA \\
\hline Age (years) & $28.9( \pm 6.1)$ & $29.1( \pm 4.4)$ & $\mathrm{p}=0.83$ \\
\hline Female gender, $\mathbf{n}$ & $7(70 \%)$ & $2(25 \%)$ & $\mathrm{p}=0.15$ \\
\hline Caucasian race, $\mathbf{n}$ & $8(80 \%)$ & $6(75 \%)$ & $\mathrm{p}=0.62$ \\
\hline
\end{tabular}




\section{Table II}

Cardiac diagnoses

List of congenital cardiac diagnosis for the sternotomy versus control groups.

\begin{tabular}{|ll|}
\hline Sternotomy, $\mathbf{n = 1 0}$ & Control, $\mathbf{n = 8}$ \\
\hline D-transposition (2) & Congenitally corrected transposition (L-TGA) (2) \\
\hline Single ventricle (3) & Patent ductus arteriosus (2) \\
\hline Atrioventricular canal & Coarctation \\
\hline Aortic stenosis & Bicuspid aortic valve \\
\hline Ventricular sepal defect & Mitral stenosis \\
\hline Pulmonary atresia/Intact ventricular septum & Congenital complete heart block \\
\hline Total anomalous pulmonary veins & \\
\hline
\end{tabular}

